\title{
Quantifying the international collaboration of researchers and research institutions
}

Pedro Cardoso ${ }^{1, \star}$, Caroline Sayuri Fukushima ${ }^{1}$, Stefano Mammola ${ }^{1,2}$

1. Laboratory for Integrative Biodiversity Research (LIBRe), Finnish Museum of Natural History Luomus, University of Helsinki, Helsinki, Finland

2. Molecular Ecology Group, Water Research Institute (IRSA), National Research Council of Italy (CNR), Verbania Pallanza, Italy

${ }^{*}$ Corresponding author: Finnish Museum of Natural History, Pohjoinen Rautatiekatu 13 (PO Box 17), 00014 University of Helsinki, Finland; pedro.cardoso@helsinki.fi

\begin{abstract}
Author-level metrics (number of publications, citations, h-index) remain prime yet controversial measures of academic performance. Among other issues, they do not account for collaboration and knowledge transfer between people, institutions and, ultimately, countries. They also do nothing to contradict the oblivion to which knowledge and data providers, such as field or lab researchers across the world, are often relegated, and they often serve to intensify the differences between high- and low-income countries. The internationalization of institutions is also gaining relevance, although no standard metrics exist yet. Given these considerations, the evaluation of careers and institutions by publication numbers does nothing to contradict the persistence of considerable inequalities within academia.
\end{abstract}

To help change the status quo, we propose the i-index, a publication metric that incentivizes the collaborative attitude of scientists across borders. Following the same rationale used to calculate the h-index, we express the i-index as the i number of co-author countries in more than i publications. Widespread use of the index may promote the quest for international collaboration beyond the limited scope of one's own country. It enhances the transfer of knowledge and recognition of often relegated collaborators. We also test the index at the institutional level, providing a tool that helps to quantify the effort many institutions are making toward fair practices in research benefits and data sharing. Our ultimate goal is that the adoption of this index will incentivize sharing and collaboration between those countries with greater access to research funding and infrastructure and less developed countries.

Keywords: academy, bibliometrics, cultural diversity, equity, inclusion, linguistic diversity, science-policy interface. 


\section{INTRODUCTION}

Scientific careers advance through a lengthy accumulation of published work (often papers) and citations. Authorship and number of publications are important criteria for making decisions about promotions and funding awards in academia (Hart and Perlis, 2021). Not without controversy (e.g., McNutt 2014; Smaldino \& McElreath 2016; Aksnes et al. 2019; Davies et al. 2021), metrics such as the number of publications, the number of citations, and derivatives (e.g., journal impact factor, h-index) have emerged as the agreed-upon measures of scientific success (Dettori et al., 2019). This evaluation of careers by numbers exerts pressure upon academics to constantly publish highly cited papers in high-impact journals, and is partly responsible for the persistence of widespread inequalities within academia (Davies et al., 2021).

To achieve maximum publication impact, the tendency is to primarily involve scholars in prestigious universities and institutions as co-authors, usually located in North America and Europe (Way et al., 2019). Researchers from the Global South are largely underrepresented in science (Maas et al. 2021). Linguistic barriers to scientific publication can also be prevalent, with work by non-Anglophone authors often being overlooked (Nuñez \& Amano 2021). This tendency is further exacerbated by the prevalence of "parachute science", the practice whereby international scientists from higher-income countries conduct studies in lower-income countries and then fly back home and finalize the research without engaging or acknowledging the help of local collaborators from those nations (Asase et al., 2021; Stefanoudis et al., 2021). This generates a vicious circle whereby authors from higher-income countries ("the Global North") can quickly and more easily boost their author-level metrics, while authors from lowincome countries ("the Global South"), belonging to minority groups or affiliated with peripheral institutions, are frequently left behind. This is a typical example of the Matthew effect "For to every one who has will more be given, and he will have abundance; but from him who has not, even what he has will be taken away" (Matthew 25:29). A strong movement is therefore emerging within academia, advocating for a shift in the current outdated system to advance science through principles of equity, diversity, and inclusivity (e.g., Trisos et al., 2020; Barber et al., 2020; Davies et al. 2021; Mori 2021).

As for individual researchers, many universities and other research institutions are pushing for both internationalization (Chan and Dimmock, 2008; de Wit and Altbach, 2021) and practices of findability, accessibility, interoperability, and reusability (FAIR) for data management and stewardship (Wilkinson et al., 2016). Benefit sharing, of not only financial gain but also knowledge, is also included in many international policies, such as the Convention on Biological Diversity (https://www.cbd.int/abs). Bearing these considerations in mind, the proportion of international collaborations in published work is set as a target for our own University of Helsinki and is part of its strategic plan. These kinds of measures and targets are probably becoming the standard in other countries and institutions. Yet they might not be enough to incentivize collaboration with larger networks and/or researchers from new countries and regions, namely those from contrasting cultures and following different scientific practices. As currently set, these measures are often achieved by focusing mostly on neighboring or more developed nations, or more prestigious institutions (Figure 1). 

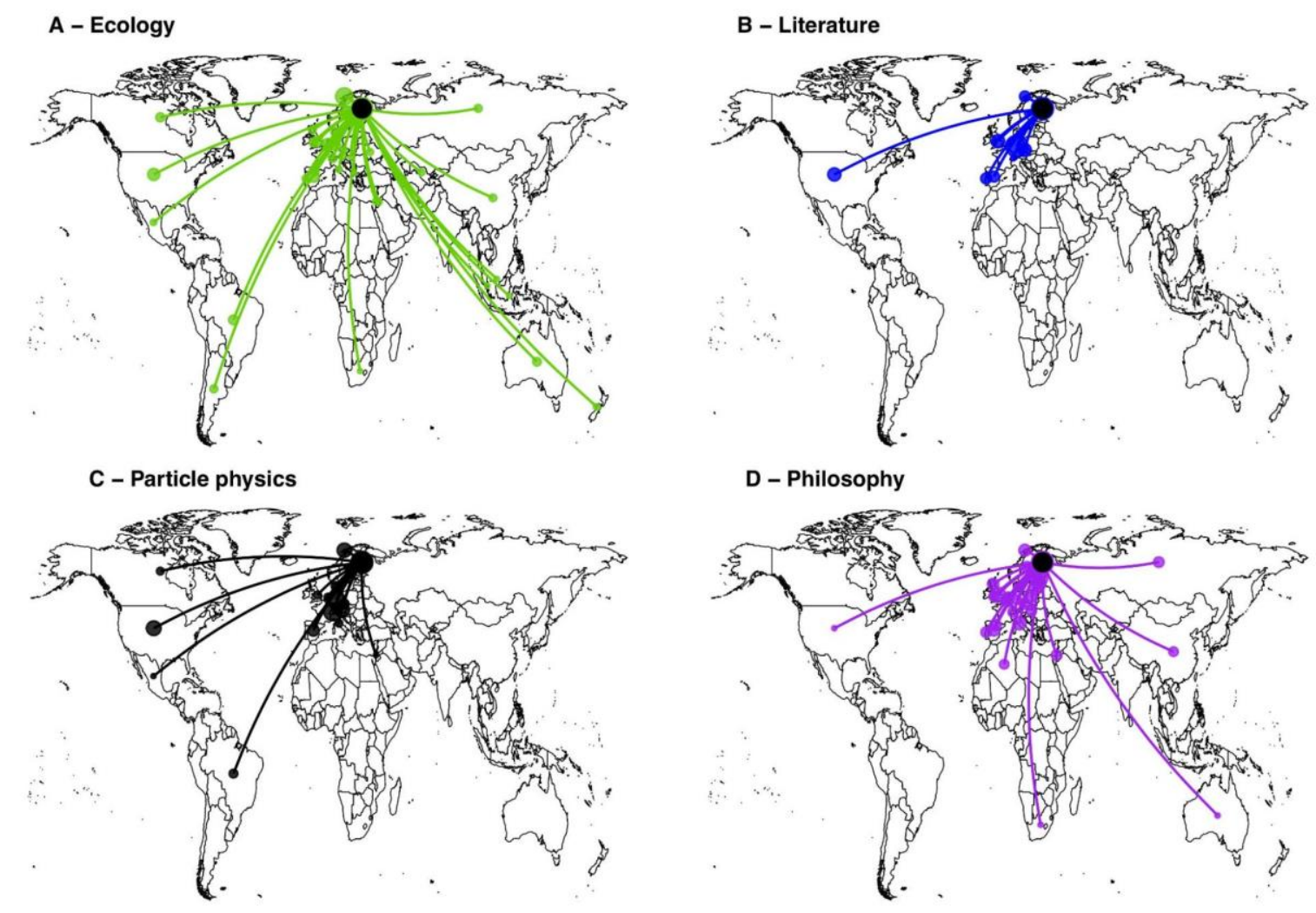

Figure 1. Collaborative network of four researchers affiliated with the University of Helsinki in the fields of Ecology (i-index $=11)(\mathbf{A})$, Literature (i-index $=3)(\mathbf{B})$, Particle physics (i-index = 6) $(\mathbf{C})$, and Philosophy (i-index = 4) (D). To generate the graphs, we selected the researcher with the highest $\mathrm{i}$-index for each discipline among the randomly sampled 200 authors. The network was generated with the RAT function i.map. The size of each dot is proportional to the number of collaborative events.

One simple way to support the change in the academic scenario would be to adopt metrics that go beyond currently adopted practices, incentivizing the collaborative attitude of scientists and institutions, especially those in higher-income countries, rather than citation and simplistic collaboration counts. To this end, we have devised the i-index, a scientometric index measuring the international collaborative attitude of scientists and institutions. In this paper, we illustrate the use of the index, introduce an R package with functions to calculate it based on the Web of Science repository of scientific literature, explore the relationship between the i-index and the h-index, and discuss some caveats in its interpretation. The adoption of this index may promote sharing and collaboration between those countries with greater access to research funding and infrastructure and less developed nations.

\section{METHODS}

\section{Index calculation}

Following the same rationale used to calculate the h-index (Hirsch, 2005), we have expressed the i-index as the i number of co-author countries in more than i publications. The five steps in this process are as follows: 
1. For each publication by a given researcher/institution, find the list of countries of the coauthors' institutions.

2. Remove duplicated countries (i.e., multiple co-authors from the same country) so as to obtain a list of unique countries.

3. Add up the number of instances per country across all publications.

4. Order countries by their total; and

5. Calculate $\mathrm{i}$ as the number of countries whose sum is higher than or equal to their rank when ordered.

As an example, a given researcher has published five papers listed in the Web of Science with the following affiliation countries:

1. $\mathrm{FI}, \mathrm{PT}, \mathrm{IT}$

2. FI, FR, NZ, PT, (PT)

3. FI, PT, IT, FR, UK, US

4. IT, FR, (FR), (FR)

5. IT, UK

Remove the duplicates (countries inside brackets), add up the values per country, and order in descending order:

IT: 4; FI: 3; PT: 3; FR: 3; UK: 2; NZ: 1; US: 1

The researcher's i-index will therefore be 3 , as three countries are listed in at least three publications.

\section{R package RAT}

We developed the $\mathrm{R}$ ( $\mathrm{R}$ core team 2020) package RAT (Research Assessment Tools; Cardoso \& Mammola 2021) to automate the calculation of the index for any researcher, group of researchers, or institution in the Web of Science (function i.index). In addition, the package allows one to visualize one's research network (function i.map) and calculates the $\mathrm{h}$-index (function h.index). The source code for the package is available in GitHub (https://github.com/cardosopmb/RAT).

\section{Data analysis}

We used the publication record of the University of Helsinki and a selection of researchers affiliated with it as a test case to explore some of the properties and trends concerning the iindex. First, we measured the evolution of the University of Helsinki's i-index across years to verify if it tends toward internationalization as written in its own strategic plan. To do so, we collected all publications by researchers affiliated with the University of Helsinki using the following Web of Science queries (search on 26 October 2021):

$$
O O=\text { Helsingin yliopisto OR OO = Helsinki University AND PY }=1990-2020
$$


In Web of Science, notation $\mathrm{OO}$ is a search for organization, and PY a search for publication year. Specifically, we extracted publications for each year between 1990 and 2020, totaling 29,521 publications.

Next, we randomly sampled 200 researchers affiliated with the University of Helsinki in 2020 across four disciplines, 50 researchers each: ecology, literature, particle physics, and philosophy. This was to verify whether collaborative practices were different between contrasting fields, with some, such as particle physics and increasingly ecology, relying on extensive networks, and others naturally being more limited in geographical range. We extracted their full publication record and calculated their i-index and $\mathrm{h}$-index. Using this database, we explored the relationship between these two indexes with a Pearson's $r$ correlation and checked whether observed patterns were consistent across disciplines.

\section{RESULTS}

The University of Helsinki's i-index slowly and steadily increased between 1990 and 2015 (data in Figshare upon acceptance). Subsequently, in 2015-2020, we observed a steep increase, with the i-index value almost tripling (from 15 to 40) (Figure 2).

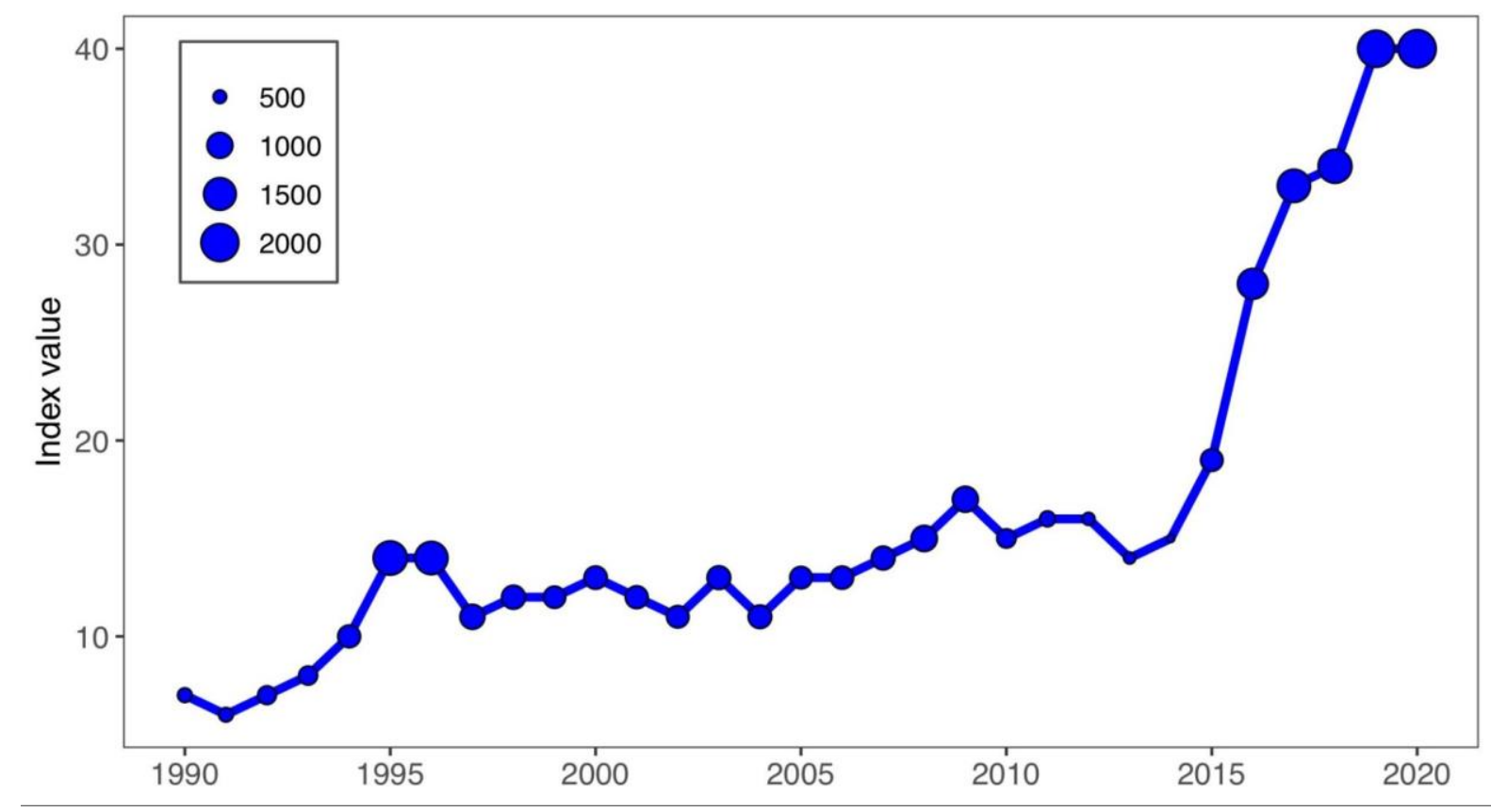

Figure 2. Temporal variation in the University of Helsinki's i-index. The temporal trend was calculated based on the publications each year between 1990 and 2020 (the dot size indicates the annual number of publications). Note the steep rise in international collaboration in recent years.

The i-index of 200 randomly sampled authors from the University of Helsinki is highly correlated with their h-index (Figure 3A). We noticed, however, substantial differences among fields (see example of collaborative networks by field in Figure 1). As expected, researchers 
working in particle physics had wider collaborative networks. This difference was not observed for ecology, however, with researchers still collaborating with co-authors from relatively few countries.
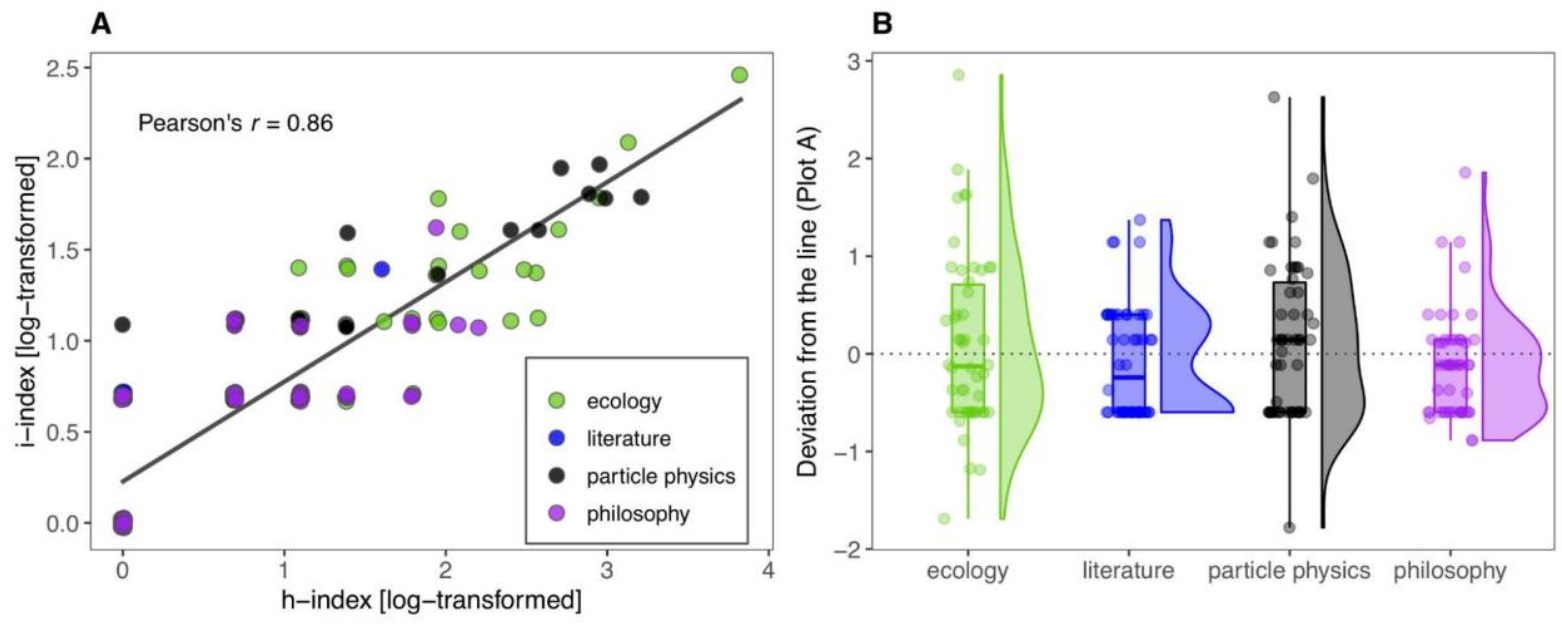

Figure 3. Correlation between the i-index and h-index. A) Relationship between h-index and i-index. Values are log-transformed and jittered to easy visualization, and colored according to the discipline. B) Boxplots and density plots illustrating positive and negative deviations from the correlation line in $\mathbf{A}$, whereby positive values indicate researchers with a higher $\mathrm{i}$ index relative to their $\mathrm{h}$-index, and vice versa.

\section{DISCUSSION}

Diversity in science primarily stems from the diversity of people. Diverse people from different cultural backgrounds bring different points of view, generate contrasting hypotheses, and devise new ways to solve old problems. This is particularly true if the international dimension is accompanied by cultural or ethnic diversity, which we hope the adoption of the i-index will indirectly promote. Some research fields are inherently collaborative across borders, and others even necessarily so. Our own research field of conservation biology is global in nature and there is now a growing tendency for recognition of the work of local partners in less developed countries (Stefanoudis et al., 2021). As an example, in the scientific journal Conservation Letters "it is a requirement that manuscripts focused on conservation in Low and Middle Income Countries include authors from the relevant country/countries" (https://conbio.onlinelibrary.wiley.com/hub/journal/1755263x/homepage/forauthors.html\#ethi cs).

Currently, the quest for international collaborators and recognizing them with co-authorship is mostly unidirectional, with researchers from the Global South seeking collaborators from the Global North, and only rarely vice versa. The reasons for this are diverse. First, it may be useful to involve researchers from the Global North simply because they have greater access to research funding and infrastructure (e.g., equipment, technologies) and not by choice. Second, referees can be biased toward authors from low-income countries (Gerwing et al., 2020), pushing these authors to involve prestigious collaborators to maximize their chances of getting published. Third, researchers from the Global South often cannot afford increasingly high publication fees (e.g., Peterson et al., 2019; Demeter and Istratii, 2020).

Although raising the level of international collaboration is ultimately in the hands of the researchers, we are aware that this process can also depend on institutional strategies and 
policies, and that shifts vis-à-vis these instruments are often necessary to change the behavior toward more internationally diverse and balanced collaborative work. The use of novel metrics in the recruitment and promotion of staff is now being proposed by several authors (Maas et al. 2021) and the i-index can provide such a tool. In addition, increasing collaboration can lead to more widespread adoption of open science practices, which follows recommendation no. 2 of the European Commission, which encourages the development of new indicators, and assesses the suitability of existing ones, to measure and support the development of open science (Wilsdon et al. 2017).

\section{Caveats in the interpretation of the i-index}

Unidimensional indexes are a blunt measure of academic value often prone to misuse; the iindex is no exception in this respect. In interpreting the i-index, one should always bear in mind important factors that may affect its calculation:

1. As with any author-level metric, comparisons in the value of the i-index among researchers should be considered in view of a number of factors, such as academic age (Kelly \& Jannions, 2006), gender (Huang et al. 2020), and parenthood status (Morgan et al., 2021).

2. The geographic location of the country of an institution may exert a non-negligible effect on the value of the i-index. For example, it can be expected that the i-index for European and African researchers that are collaborative within the boundaries of their continent will be slightly inflated simply because Europe and Africa are fragmented into a number of smaller countries. The same effect would not be visible, for example, for researchers that are collaborative within US borders.

3. The recent increase in multi-author publications can artificially inflate the index value, especially for authors that are part of broad research networks. This effect may be particularly important for certain disciplines, such as particle physics (e.g., Castelvecchi et al., 2015). In this respect, authorship status also entails different practices across disciplines.

4. The calculation of the index is (inevitably) based on authors' affiliations rather than the country of origin. On the one hand, this means that by involving authors with multiple affiliations in a publication, one can inflate the index. On the other hand, authorships may be diverse in the sense of involving authors with different nationalities, but this will not be reflected in the index if they are affiliated to institutions from the same countries.

5. Diversity of countries might not correspond to other aspects of diversity in science. For example, white scholars are often disproportionately represented in faculty (https://www.pewresearch.org/fact-tank/2019/07/31/us-college-faculty-student-diversity/).

6. While the i-index should theoretically range from 1 to 195 - the current number of countries worldwide - this value can fluctuate as new countries are established and others disappear. For example, when a researcher's publication record spans multiple decades, countries that do not exist anymore (e.g., USSR) will count toward the i-index value.

7. Authors affiliated with small countries such as Vatican City, San Marino, Andorra, and small oceanic islands (Palau, Marshall Islands, etc.) may become highly requested coauthors in the quest for maximizing the i-index. Predictably, such scholars will find their email inboxes full of "Requests for collaboration", and hence we recommend adopting antispam filters when appropriate. 


\section{Conclusions}

Applied at both researcher and institution levels, we expect the i-index to enhance the transfer of knowledge among countries and the recognition of often relegated collaborators and institutions, rather than being another metric that leads to more exclusion or promotes more discriminatory rewards in science. On the contrary, we hope that increasing collaboration and the transfer of knowledge and financial and human resources between countries helps reduce inequalities in science. The recent wide adoption of new ways to communicate and collaborate in science, with many meetings and conferences moving online, presents a good opportunity to foster international collaboration with researchers that until today did not have the means to attend relevant events.

In combination with other indices, such as the h-index and Altmetrics, the i-index might provide a better way to evaluate the performance of a researcher. It adds to the diversity component of evaluations or analyses, even if it does not resolve, or intend to resolve, other biases in science. Finally, the i-index provides a tool that helps to quantify the efforts many institutions are expending toward fair practices in relation to research benefits and data sharing.

\section{Acknowledgments}

PC and CSF are supported by Kone Foundation. SM is supported by the European Commission through Horizon 2020 Marie Skłodowska-Curie Actions (MSCA) individual fellowships (Grant no. 882221). Ricardo Rocha and Diogo Verissimo commented on a preliminary draft.

\section{Author contribution}

PC conceptualized the index. PC and SM prepared the R package code and performed analyses. All authors contributed to the writing.

\section{Literature cited}

Aksnes, D.W., Langfeldt, L. \& Wouters, P. (2019). Citations, citation indicators, and research quality: an overview of basic concepts and theories. SAGE Open, 9: 2158244019829575. https://doi.org/10.1177/2158244019829575

Asase, A., Mzumara-Gawa, T.I., Owino, J.O., Peterson, A.T. \& Saupe, E. (2021). Replacing "parachute science" with "global science" in ecology and conservation biology. Conservation Science and Practice, e517. https://doi.org/10.1111/csp2.517

Barber, P.H., Hayes, T.B., Johnson, T.L. \& Márquez-Magaña, L. (2020). Systemic racism in higher education. Science, 369: 1440-1441. https://doi.org/10.1126/science.abd7140

Cardoso, P. \& Mammola, S. (2021). RAT: Research Assessment Tools. Available online at: https://CRAN.R-project.org/package=RAT 
Castelvecchi, D. (2015). Physics paper sets record with more than 5,000 authors. Nature News, available online at: https://doi.org/10.1038/nature.2015.17567

Chan, W.W.Y. \& Dimmock, C. (2008). The internationalization of universities: Globalist, internationalist and translocalist models. Journal of Research in International Education, 7: 184-204. https://doi.org/10.1177/1475240908091304

Davies, S.W., et al. (2021). Promoting inclusive metrics of success and impact to dismantle a discriminatory reward system in science. PLOS Biology, 19: e3001282. https://doi.org/10.1371/journal.pbio.3001282

Dettori, J.R., Norvell, D.C. \& Chapman, J.R. (2019). Measuring academic success: the art and science of publication metrics. Global Spine Journal, 9: 243-246. https://doi.org/10.1177/2192568219831003

Gerwing, T.G., Gerwing, A.M.A., Avery-Gomm, S., Choi, C.Y., Clements, J.C. \& Rash, J.A. (2020). Quantifying professionalism in peer review. Research Integrity and Peer Review, 5: 9. https://doi.org/10.1186/s41073-020-00096-x

de Wit, H. \& Altbach, P.G. (2021). Internationalization in higher education:global trends and recommendations for its future, Policy Reviews in Higher Education, 5:1, 28-46. https://doi.org/10.1080/23322969.2020.1820898

Hart, K.L. \& Perlis, R.H. (2021). Authorship inequality: a bibliometric study of the concentration of authorship among a diminishing number of individuals in high impact medical journals 2008-2019. BMJ Open, 11: e046002. https://doi.org/10.1136/bmjopen-2020-046002

Hirsch, J.E. (2005). An index to quantify an individual's scientific research output. Proceedings of the National Academy of Sciences, 102: 16569-16572. https://doi.org/10.1073/pnas.0507655102

Huang, J., Gates, A.J., Sinatra, R. \& Barabási, A.-L. (2020). Historical comparison of gender inequality in scientific careers across countries and disciplines. Proceedings of the National Academy of Sciences, 117: 4609-4616. https://doi.org/10.1073/pnas.1914221117

Kelly, C.D. \& Jennions, M.D. (2006). The $\mathrm{h}$ index and career assessment by numbers. Trends in Ecology \& Evolution, 21: 167-170. https://doi.org/10.1016/j.tree.2006.01.005

Demeter, M. \& Istratii, R. (2021). Scrutinising what open access journals mean for global inequalities. Research Quarterly, 36: 505-522. https://doi.org/10.1007/s12109-020-09771-9

Maas, B., Pakeman, R.J., Godet, L., Smith, L., Devictor, V. \& Primack, R. (2021). Women and Global South strikingly underrepresented among top-publishing ecologists. Conservation Letters, 14: e12797. https://doi.org/10.1111/conl.12797

McNutt, M. (2014). The measure of research merit. Science, 346: 1155. https://doi.org/10.1126/science.aaa3796

Morgan, A.C. et al. (2021). The unequal impact of parenthood in academia. Science Advances, 7: eabd1996. https://doi.org/10.1126/sciadv.abd1996

Mori, A.S. (2021). Diversity, equity, and inclusion in academia to guide society. Trends in Ecology and Evolution, in press. https://doi.org/10.1016/j.tree.2021.10.010 
Nuñez, M.A. \& Amano, T. (2021). Monolingual searches can limit and bias results in global literature reviews. Nature Ecology \& Evolution, 5: 264. https://doi.org/10.1038/s41559-02001369-w

Peterson, A.T., et al. (2019). Open access solutions for biodiversity journals: Do not replace one problem with another. Diversity and Distributions, 25: 5-8. https://doi.org/10.1111/ddi.12888

R Core Team (2020). R: A language and environment for statistical computing. R Foundation for Statistical Computing. Available online from www.R-project.org

Smaldino, P.E. \& McElreath, R. (2016). The natural selection of bad science. Royal Society Open Science, 3: 160384. https://doi.org/10.1098/rsos.160384

Stefanoudis, P.V., Licuanan, W.Y., Morrison, T.H., Talma, S., Veitayaki, J. \& Woodall, L.C. (2021). Turning the tide of parachute science. Current Biology, 31: 184-185. https://doi.org/10.1016/j.cub.2021.01.029

Trisos, C.H., Auerbach, J. \& Katti, M. (2021). Decoloniality and anti-oppressive practices for a more ethical ecology. Nature Ecology \& Evolution, 5: 1205-1212. https://doi.org/10.1038/s41559-021-01460-w

Way, S.F., Morgan, A.C., Larremore, D.B. \& Clauset, A. (2019). Productivity, prominence, and the effects of academic environment. Proceedings of the National Academy of Sciences, 116: 10729-10733. https://doi.org/10.1073/pnas.1817431116

Wilkinson, M.D., et al. (2016). The FAIR Guiding Principles for scientific data management and stewardship. Scientific data, 3: 1-9. https://doi.org/10.1038/sdata.2016.18 